\title{
Nonlinear Inverse Perturbation Method in Dynamic Analysis
}

\author{
Ki-Ook Kim, ${ }^{*}$ William J. Anderson, $†$ and Robert E. Sandstrom $\ddagger$ \\ The University of Michigan, Ann Arbor, Michigan
}

\begin{abstract}
An analytical method is presented for the automated redesign of the modal characteristics of undamped mechanical systems. The method is based on a perturbation of the eigensystem, and all nonlinear terms are carried so that large changes can be considered. The user requests frequency and mode shape changes. The structural changes are expressed through design parameters $\alpha_{i}$ representing conventional thicknesses, moments of inertia, etc. A penalty function method is used in which the original objective function is a minimum weight condition and the penalty term is a properly normalized set of residual nodal force errors. Solutions converge well for typical example problems using five cycles of the conjugate gradient method. The method is superior to linear methods, which have shown error when significant mode shape changes are required. It is believed to be computationally superior to series solutions, such as Taylor's series, when only one or two frequencies and mode shapes are of interest. In addition, the method is not subject to a limited radius of convergence in the design variables as is encountered in series solutions.
\end{abstract}

\section{Nomenclature}

$\left[C_{1}\right]-\left[C_{3}\right]$

$F($ )

$f()$

$h$

$[k],\left[k^{\prime}\right]$

$[K],\left[K^{\prime}\right]$

$[\bar{K}]_{e},[\bar{K}]_{e}$

$[\Delta k],\left[\Delta k_{e}\right]$

$[m],\left[m^{\prime}\right]$

$[M],\left[M_{-}^{\prime}\right]$

$[\bar{M}]_{e},[\bar{M}]_{e}$

$[\Delta m],\left[\Delta m_{e}\right]$

$L$

$M$

$N$

$P($ )

$\{R\}$

$X$

$X_{0}, X_{k}$

$\alpha_{e}^{k}, \alpha_{e}^{m}$

$\alpha_{e}$

$\alpha_{\lambda}$

[Г]

$\nabla$

$[\lambda],\left[\lambda^{\prime}\right]$

$[\Delta \lambda]$

$\mu$

$[\phi],\left[\phi^{\prime}\right]$

function method turbed system, $(\leq N)$

$=$ penalty term problem and mass changes element $e$ $\Delta \lambda / \lambda$

$=$ gradient operator and perturbed

$=$ penalty parameter
$\{\Delta \phi\},\{\Delta \phi\}$

$=$ coefficient matrices in Eq. (20)

$=$ modified objective function in penalty

$=$ original objective function

$=$ thickness of cantilever beam

$=$ assembled stiffness, original and perturbed

= generalized stiffness, original and per-

$=$ element coefficient matrices in Eq. (20)

$=$ changes in stiffness in system and elements

$=$ assembled mass, original and perturbed

$=$ generalized mass, original and perturbed

$=$ element coefficient matrices in Eq. (20)

$=$ changes in mass, in system and elements

$=$ number of elements to be perturbed

$=$ number of modes used in $N$ dimensional

$=$ dimension of dynamic system

$=$ residual error in force equilibrium

$=$ vector of variables in minimization

$=$ starting point and $k$ th iteration point

$=$ design variables of element $e$ in stiffness

$=\Delta h_{e} / h_{e}$ fractional thickness change in

$=$ dimensionless frequency design variable,

$=$ normalization matrix, $N \times N$

= diagonal matrix of eigenvalues, original

$=$ eigenvalue perturbation

$=$ modal matrix, original and perturbed

$=$ specified and unspecified mode shape changes

Received July 19,1982; revision received Dec. 7, 1982. Copyright (C 1983 by William J. Anderson. Published by the American Institute of Aeronautics and Astronautics with permission.

${ }^{*}$ Graduate Student, Aerospace Engineering. Student Member AIAA.

†Professor, Aerospace Engineering. Member AIAA.

$\$$ Adjunct Assistant Professor, Naval Architecture and Marine Engineering (presently, Research Engineer, Exxon Production

Research Co., Houston, Texas). Member AIAA.
Superscripts

$\begin{array}{ll}{[]^{T}} & =\text { transpose of a matrix } \\ {[]^{-1}} & =\text { inverse of a matrix }\end{array}$

\section{Introduction}

$\mathbf{M}$ ODAL analysis of complex structures is often performed with general-purpose finite element codes. When the results show that natural frequencies and mode shapes need to be changed, it is usually not clear how to modify the original design to achieve the desired goals. In one such code, MSC/NASTRAN, one can extract stiffnesses, masses, frequencies, and mode shapes through the use of the direct matrix abstraction program (DMAP). The information from a baseline structure can then be used in a perturbation procedure to allow redesign.

The procedure discussed here was motivated by the linear perturbation work by Stetson et al. ${ }^{1-4}$ Examination of the linear method has led the authors to include all nonlinear terms in the perturbation equations. It was discovered that one second-order term is as large as first-order terms when typical redesign problems are solved. Neglect of second-order terms can lead to unacceptable error, particularly in mode shape redesign.

Nonlinear mathematical programming is used to solve the perturbation problem. The system is usually underdetermined, i.e., there is more than one physical redesign that will meet the dynamic goals. To achieve a unique redesign, one requires either minimum weight or the least structural change from the original design.

The procedure to be developed is straightforward. It involves only a single finite element analysis of a baseline structure. Subsequent mathematical programming involves a smaller number of variables. It is believed that this approach offers computational advantages over competing methods for redesign cases that involve only several frequencies and modes.

The method developed here is similar in spirit to the work of Pierson, ${ }^{5}$ in which optimal control ideas are used in minimum weight design with frequency constraints. The present work differs from Pierson's in the use of a perturbation procedure and application to the finite element method. The perturbation scheme in turn leads to new possibilities in mode shape redesign and to differences in the penalty function. For a general discussion of structural synthesis and optimization, the reader is referred to excellent reviews by Schmit $^{6}$ and Vanderplaats. ${ }^{7}$

Finally, the method can also be used in model correlation and structural system parameter identification studies. In 
either case, if the frequencies and mode shapes to be matched are given, the method is attractive because of its simplicity and the reduced number of unknown variables.

\section{Inverse Perturbation}

The $N$ dimensional eigenvalue problem is written in compact form as

$$
[k][\phi]=[m][\phi][\lambda]
$$

All matrices are $N \times N$ in size. Premultiplying Eq. (1) by [ $\phi]^{T}$ yields an energy balance,

where

$$
[K]=[M][\lambda]
$$

$$
\begin{gathered}
{[K]=[\phi]^{T}[k][\phi]} \\
{[M]=[\phi]^{T}[m][\phi]}
\end{gathered}
$$

If this baseline system is perturbed, one obtains

$$
\left[k^{\prime}\right]\left[\phi^{\prime}\right]=\left[m^{\prime}\right]\left[\phi^{\prime}\right]\left[\lambda^{\prime}\right]
$$

To show the nonlinear character of the perturbed equation of motion, it is rewritten as

$$
([k]+[\Delta k])([\phi]+[\Delta \phi])=([m]+[\Delta m])([\phi]+[\Delta \phi])([\lambda]+[\Delta \lambda])
$$

where terms up to third order are present. This is not a uniform expansion of dependent variables in terms of small parameters, but is an implicit relation between the independent and dependent perturbed quantities. The perturbation terms are therefore not well ordered. To show the relative sizes of the terms and the errors in dropping higher terms, a small physical problem is documented in the Appendix. It is concluded that the $[\Delta k][\Delta \phi]$ term is numerically large and should not be neglected in typical design problems. All of the terms in Eq. (5b) are included in subsequent work done here.

Another approach to the perturbation problem would have been to uncouple Eqs. (5) by premultiplying by $\left[\phi^{\prime}\right]^{T}$ to yield

$$
\left[K^{\prime}\right]=\left[M^{\prime}\right]\left[\lambda^{\prime}\right]
$$

Stetson et al. ${ }^{1-4}$ used a linearized version of Eq. (6) in their perturbation method. An advantage of that method is the uncoupling of the frequency and mode shape changes. For large sets of equations, it is necessary to use a truncated set of modes in the method. In this case, Eq. (6) suffers from a mild drawback in that the truncated energy equations are only a necessary and not a sufficient condition for solution. ${ }^{8}$ Also, the linear perturbation does not guarantee symmetry or even the diagonality of the matrices in Eq. (6). For frequency goals, the linear energy formulation gives good accuracy ${ }^{9}$; however, it is often not accurate for significant mode shape redesign.

The current work puts emphasis on mode shape changes rather than frequency changes. For convenience, consider a single mode,

$$
\left[k^{\prime}\right]\left\{\phi^{\prime}\right\}_{i}=\left[m^{\prime}\right]\left\{\phi^{\prime}\right\}_{i} \lambda_{i}^{\prime}
$$

Specification of the desired goals $\left\{\phi^{\prime}\right\}_{i}, \lambda_{i}^{\prime}$ can be done in several ways. In practice, all degrees of freedom in $\left\{\phi^{\prime}\right\}_{i}$ cannot be arbitrarily specified. Geometric boundary conditions and normalization of $\left\{\phi^{\prime}\right\}_{i}$ must be observed; the remaining values are free. The perturbed mode shape is expressed as

$$
\{\Delta \phi\}_{i}=\left\{\begin{array}{c}
\Delta \dot{\phi} \\
\Delta \bar{\phi}
\end{array}\right\}_{i}
$$

where $\left\{\Delta_{\phi}^{\circ}\right\}_{i}$ and $\{\Delta \bar{\phi}\}_{i}$ are the specified and the unspecified degrees of freedom, respectively.

The structural change can be decomposed into $L$ element changes,

$$
\begin{aligned}
& {[\Delta k]_{\text {system }}=\sum_{e=1}^{L}\left[\Delta k_{e}\right]} \\
& {[\Delta m]_{\text {system }}=\sum_{e=1}^{L}\left[\Delta m_{e}\right]}
\end{aligned}
$$

Furthermore, each element change can be expressed as a fractional change from the original system (or a sum of terms as needed to separate bending, stretching, torsion, etc.), as

$$
\begin{gathered}
{\left[\Delta k_{e}\right]=\left[k_{e}\right] \alpha_{e}^{k}} \\
{\left[\Delta m_{e}\right]=\left[m_{e}\right] \alpha_{e}^{m}}
\end{gathered}
$$

These relations may be linear or nonlinear.

Substituting Eqs. (8-11) into Eq. (7), one obtains a residual force error

$$
\{R\} \equiv\left[k^{\prime}\right]\left\{\phi^{\prime}\right\}_{i}-\left[m^{\prime}\right]\left\{\phi^{\prime}\right\}_{i} \lambda_{i}^{\prime}=0
$$

where $\{R\}$ is a function of the design variables, unspecified degrees of freedom, and frequency change. Equation (12) may be underdetermined, uniquely posed, or overdetermined: The present paper considers only the underdetermined equation.

\section{Solution by Mathematical Programming}

In the case of an underdetermined problem, mathematical programming techniques ${ }^{10-12}$ can be used to achieve minimum weight or minimum change of the structure with Eq. (12) as the equality constraint. The penalty function method gives the modified objective function,

$$
F\left(\{\alpha\},\{\Delta \bar{\phi}\}_{i}, \Delta \lambda_{i}\right)=f(\{\alpha\})+\mu P\left(\{\alpha\},\{\Delta \bar{\phi}\}_{i}, \Delta \lambda_{i}\right)
$$

where $f$ is the original objective function, $\mu$ the penalty parameter, and $P$ the penalty term containing the equilibrium error [Eq. (12)]. The penalty term is temporarily chosen as

$$
P(X) \equiv\{R\}^{T}\{R\}
$$

where $X$ is the vector of variables $\{\alpha\},\{\Delta \bar{\phi}\}_{i}$, and $\Delta \lambda_{i}$.

In practice, normalization procedures allow better numerical behavior. Indeed, sample problems have shown that structural elements such as beams are dominated by bending moment errors at the expense of shearing forces. Appropriate scaling is required. A general normalization is written $^{12}$

$$
P(X)=\{R\}^{T}[\Gamma]\{R\}
$$

The best choice for the $k$ th iteration would be

$$
[\Gamma]=\left\{\left[\nabla R\left(X_{k}\right)\right]\left[\nabla R\left(X_{k}\right)\right]^{T}\right\}^{-1}
$$

However, $[\Gamma]$ is expensive to calculate at each step, and can be approximated by

$$
\left[\Gamma_{0}\right]=\left\{\left[\nabla R\left(X_{0}\right)\right]\left[\nabla R\left(X_{0}\right)\right]^{T}\right\}^{-1}
$$

where $X_{0}$ is the initial point of the iteration. A simpler way of normalization is to use the new mode shape $\left\{\phi^{\prime}\right\}_{i}$. In this case, $[\Gamma]$ is diagonal,

$$
\Gamma_{j j}=\phi_{j i}^{2}
$$


With this normalization, the penalty function acts to minimize the energy error in each degree of freedom.

The magnitude of the penalty parameter $\mu$ dictates relative emphasis on accurate equilibrium vs accurate weight minimization (or least change). Numerical examples are needed to study both this and the normalization procedure.

If the value of $\mu$ is large, the penalty term will dominate the search pattern and the algorithm tends to go toward the equilibrium state rather than the minimum value of the original objective function. If $\mu$ is small, much emphasis is put on the minimization of the original objective function and one may not obtain accurate equilibrium (physical) results. In sample problems, the initial values of $\mu$ are chosen so that the total penalty $(\mu P)$ may be $100 \sim 1000$ times greater than the values of the original objective function. As in the sequential unconstrained minimization technique, $\mu$ is increased at each cycle by a factor of 10 .

For minimum weight, which is considered in this paper, the objective function is

$$
f\left(\alpha_{e}\right)=\sum_{e=1}^{L} \alpha_{e}
$$

Any desired inequality constraints are eliminated by changing variables through a sine transformation. For instance, in the case of large change, $\alpha_{e}$ must be algebraically greater than -1.0 so that the element does not vanish. The partial conjugate gradient method of Fletcher-Reeves in the IBM Scientific Subroutine Package ${ }^{13}$ is used for the unconstrained minimization. This method allows an initial guess at the solution or defaults to the baseline structure $\left(\alpha_{e}=0, e=1, L\right.$; $\left.\{\Delta \bar{\phi}\}_{i}=0\right)$.

\section{Numerical Examples}

Flexural vibration of a uniform, cantilever beam (Fig. 1) is used to illustrate the method. To simplify the problem, the beam cross section is rectangular, the motion will be planar, and shear deformation and axial displacement will not be included. After eliminating the 2 degrees of freedom at the wall, one has 10 elastic degrees of freedom. The set of included modes, found by the inverse power method in MSC/NASTRAN, is arbitrarily limited to five; hence, the examples include truncation effects.

The desired design changes will be confined to the third mode, although multiple mode changes (or constraints) can be accommodated in Eq. (13) by including equilibrium terms relevant to each such mode. In the following examples, both frequency and a modal deflection are specified for the third mode. The remaining modes are allowed to "drift" without constraint. In a practical problem, one might also constrain several other frequencies and mode shapes that are important.

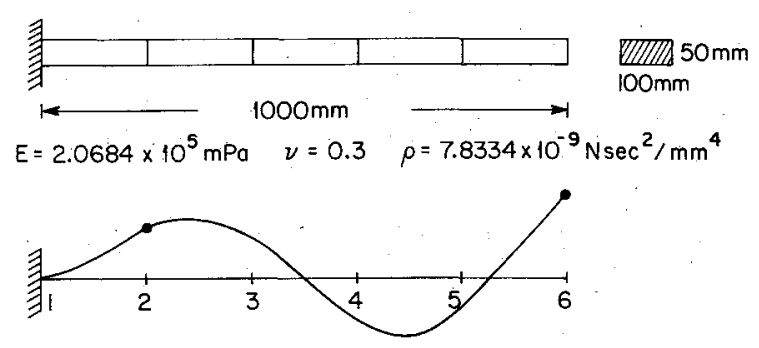

$\omega_{3}^{2}=2.109125 \times 10^{7} \mathrm{rad}^{2} / \mathrm{sec}^{2} \quad \phi 33=0.6048298 \quad \phi 11,3=1.0$

Fig. 1 Baseline cantilever beam in flexural vibration, third mode.

A common goal of each redesign will be to change mode 3 so that transverse displacement at node 2 is 0.5174698 instead of the baseline value (Fig. 1) of 0.6048298 . The mode will be normalized so that tip displacement is $\phi_{11,3}$ always unity. Different frequency changes $\Delta \lambda_{3}$ will be given in each case.

The design variables are the thicknesses of the five beam elements. The width remains constant. The stiffness matrix, mass matrix, frequencies, and mode shapes are extracted from an MSC/NASTRAN run of the baseline structure through DMAP ALTER and OUTPUT4. The matrix manipulations were done with DMAP statements; however, for the mathematical programming, a FORTRAN program was used as a postprocessor.

One can substitute the specified values into the residual force equation (12) and rearrange to obtain the general form,

$$
\begin{aligned}
& \{R\}=\sum_{e=1}^{L}\left\{[\bar{K}]_{e}\left(3 \alpha_{e}+3 \alpha_{e}^{2}+\alpha_{e}^{3}\right)+[\bar{M}]_{e} \alpha_{e}\left(1+\alpha_{\lambda}\right)\right\} \\
& +\left\{\sum_{e=1}^{L}\left([\overline{\bar{K}}]_{e}\left(3 \alpha_{e}+3 \alpha_{e}^{2}+\alpha_{e}^{3}\right)+[\overline{\bar{M}}]_{e} \lambda\left(I+\alpha_{\lambda}\right) \alpha_{e}\right)\right\}\{\Delta \bar{\phi}\} \\
& \quad+\left\{C_{0}\right\}+\left[C_{l}\right]\{\Delta \bar{\phi}\}+\left[C_{2}\right] \alpha_{\lambda}+\left[C_{3}\right]\{\Delta \bar{\phi}\} \alpha_{\lambda}
\end{aligned}
$$

where $\alpha_{\lambda} \equiv \Delta \lambda / \lambda$ and the coefficient matrices are obtained from the baseline structure and the specified values in the

\begin{tabular}{|c|c|c|c|c|c|c|}
\hline & Baseline & 1 & 2 & 3 & 4 & Goal \\
\hline Type of normalization & 一 & None & $\begin{array}{c}\phi^{\prime} \\
\text { Eq. (18) }\end{array}$ & $\begin{array}{c}\phi^{\prime} \\
\text { Eq. (18) }\end{array}$ & $\begin{array}{c}\Gamma_{0} \\
\text { Eq. (17) }\end{array}$ & $\overline{-}$ \\
\hline $\begin{array}{l}\text { Approximate starting } \\
\text { point }\end{array}$ & - & Yes & No & Yes & Yes & - \\
\hline $\begin{array}{l}\text { Perturbation } \\
\alpha_{1}=\Delta h_{1} / h_{1} \\
\alpha_{2} \\
\alpha_{3} \\
\alpha_{4} \\
\alpha_{5} \\
\Sigma \alpha_{i}\end{array}$ & $\begin{array}{l}0 \\
0 \\
0 \\
0 \\
0 \\
0\end{array}$ & $\begin{array}{r}0.1379 \\
-0.0506 \\
-0.0737 \\
-0.1008 \\
-0.0314 \\
-0.1186 \\
\end{array}$ & $\begin{array}{r}0.0952 \\
-0.0116 \\
-0.0114 \\
-0.0339 \\
-0.1068 \\
-0.0559 \\
\end{array}$ & $\begin{array}{r}0.1915 \\
-0.0289 \\
-0.0901 \\
-0.0634 \\
-0.0827 \\
-0.0735 \\
\end{array}$ & $\begin{array}{r}0.1675 \\
-0.0151 \\
-0.0623 \\
-0.0620 \\
-0.0841 \\
-0.0560 \\
\end{array}$ & $\begin{array}{c}- \\
- \\
- \\
- \\
\text { (Minimum) }\end{array}$ \\
\hline$\omega_{3}^{\frac{1}{33} \times 10^{-7}}$ & $\begin{array}{c}0.6048298 \\
2.109125\end{array}$ & $\begin{array}{l}0.5656018 \\
1.982179\end{array}$ & $\begin{array}{l}0.5174651 \\
2.109110\end{array}$ & $\begin{array}{l}0.5174702 \\
2.109126\end{array}$ & $\begin{array}{l}0.5174689 \\
2.109122\end{array}$ & $\begin{array}{l}0.5174698 \\
2.109125\end{array}$ \\
\hline
\end{tabular}

Table 2 Case 1: Frequencies of perturbed beam (subcase 3)

\begin{tabular}{lccccc}
\hline \hline Mode & 1 & 2 & 3 & 4 & 5 \\
\hline$\omega \times 10^{-2}$ & 2.6079 & 16.351 & 45.925 & 90.725 & 150.58 \\
$\omega^{\prime} \times 10^{-2}$ & 3.0009 & 16.943 & 45.925 & 88.630 & 147.21 \\
$\Delta \omega / \omega, \%$ & 15.07 & 3.62 & 0.00 & -2.31 & -2.24 \\
\hline \hline
\end{tabular}

Table 1 Case 1: Third modal frequency constrained 
perturbed eigenvector. The penalty function and the gradients are obtained from this equation. In the gradient evaluation, much effort is saved because calculations from the function evaluation are reused. (The coefficient matrices are not defined here because of their complexity.)

All of the solutions use five cycles in the conjugate gradient method. Convergence observations are hence limited to a comparison of the accuracy of different normalizations and starting points at the same number of steps taken.

\section{Case 1: Mode Changed, Frequency Fixed}

Consider the standard mode change (defined above) in conjunction with a constrained third natural frequency:

$$
\begin{gathered}
\Delta \phi_{33}=-0.08736 \quad(-14.4 \%) \\
\Delta \lambda_{3} / \lambda_{3}=0
\end{gathered}
$$

The other mode shapes and frequencies are unconstrained.

Four redesigns (subcases) are presented. They differ in the normalization procedure and in whether or not an approximate starting point in the variables $\alpha_{e}$ and $\{\Delta \phi\}_{i}$ is given by the user. An approximate starting point can be determined from the linear theory. ${ }^{9}$

The results (Table 1) show that the two types of normalization given in Eqs. (17) and (18) yield more accurate results than the unnormalized case. The use of an approximate starting point rather than the baseline structure improves the solution search and leads to the minimum weight design among the three normalized subcases.

The boxed outline in Table 1 represents the results of reanalysis done with MSC/NASTRAN. In this analysis, the perturbed thicknesses obtained through mathematical programming were used.

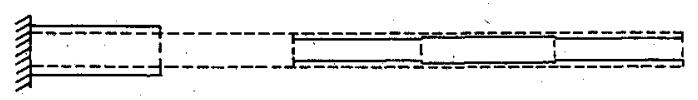

Fig. 2 Case 1 (subcase 3): redesign for change in third-mode shape and fixed frequency (thickness change is exaggerated by a factor of two).

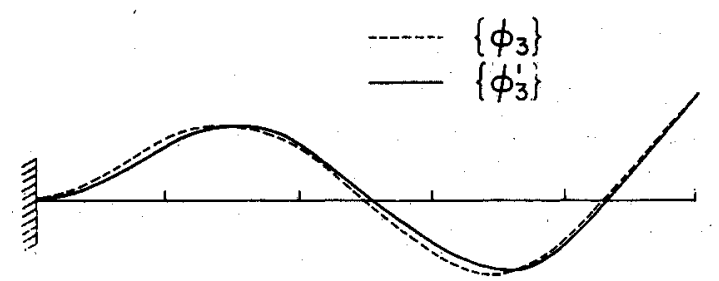

Fig. 3 Case 1 (subcase 3): third-mode shape of perturbed beam.
The redesigned structure is shown in Fig. 2. Reanalysis of this perturbed structure yields the third mode shape shown in Fig. 3 and the frequencies in Table 2. There is some drift of the unconstrained frequencies.

\section{Case 2: Mode Changed, 30\% Frequency Change}

Consider both a frequency and a mode change:

$$
\begin{aligned}
\Delta \phi_{33} & =-0.08736 \quad(-14.4 \%) \\
\Delta \lambda_{3} / \lambda_{3} & =0.69000 \quad(+30 \% \text { in frequency })
\end{aligned}
$$

The results (Table 3 ) indicate that excellent frequency and mode shape accuracy is obtained with either of the normalization procedures. The use of an approximate starting point leads to a slight improvement in accuracy at the fivecycle level of solution shown here.

The solution where no normalization is used provides the best minimum weight solution, but at the expense of accuracy in eigenvalue and eigenvector. In addition, the frequency change obtained (as confirmed by reanalysis) is only $30 \%$ of that desired, so it appears that a normalization procedure must be utilized.

The redesigned structure is given in Fig. 4. Reanalysis of the perturbed structure gives the mode shape in Fig. 5 and the frequencies in Table 4 . The unconstrained frequencies tend to

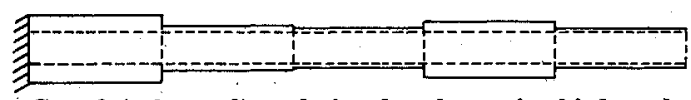

Fig. 4 Case 2 (subcase 3): redesign for change in third-mode shape and $30 \%$ increase in third frequency (thickness change is exaggerated by a factor of two).

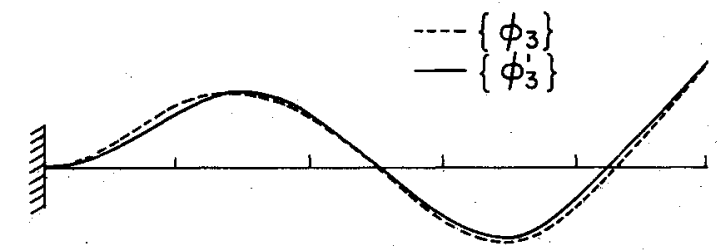

Fig. 5 Case 2 (subcase 3): third-mode shape of perturbed beam.

\begin{tabular}{|c|c|c|c|c|c|c|}
\hline & Baseline & 1 & 2 & 3 & 4 & Goal \\
\hline Type of normalization & - & None & $\phi^{\prime}$ & $\phi^{\prime}$ & $\Gamma_{0}$ & - \\
\hline $\begin{array}{l}\text { Approximate starting } \\
\text { point }\end{array}$ & - & Yes & No & Yes & Yes & - \\
\hline \multicolumn{7}{|l|}{ Perturbation } \\
\hline$\alpha_{l}=\Delta h_{l} / h_{l}$ & 0 & 0.3433 & 0.4032 & 0.4920 & 0.4904 & - \\
\hline$\alpha_{2}$ & 0 & 0.1085 & 0.1980 & 0.1638 & 0.1490 & - \\
\hline$\alpha_{3}$ & 0 & 0.0358 & 0.1503 & 0.1092 & 0.1587 & - \\
\hline$\alpha_{4}$ & 0 & -0.0270 & 0.3592 & 0.3304 & 0.3230 & - \\
\hline$\alpha_{5}$ & 0 & -0.0271 & 0.0995 & 0.1038 & 0.1049 & - \\
\hline$\Sigma \alpha_{i}$ & 0 & 0.4904 & 1.2104 & 1.1991 & 1.2261 & (Minimum) \\
\hline$\phi_{33}$ & 0.6048298 & 0.4825762 & 0.5173326 & 0.5174707 & 0.5174723 & 0.5174698 \\
\hline$\omega_{3}^{2} \times 10^{-7}$ & 2.109125 & 2.618730 & 3.563850 & 3.564454 & 3.564451 & 3.564421 \\
\hline
\end{tabular}

Table 4 Case 2: Frequencies of perturbed beam (subcase 3)

\begin{tabular}{lccccc}
\hline \hline Mode & 1 & 2 & 3 & 4 & 5 \\
\hline$\omega \times 10^{-2}$ & 2.6079 & 16.351 & 45.925 & 90.725 & 150.58 \\
$\omega^{\prime} \times 10^{-2}$ & 3.6321 & 21.734 & 59.703 & 110.91 & 183.54 \\
$\Delta \omega / \omega, \%$ & 39.27 & 32.92 & 30.00 & 22.25 & 21.89 \\
\hline \hline
\end{tabular}

Table 3 Case 2: Third modal frequency increased $30 \%$ 
Table 5 Case 3: Third modal frequency increased 200\%

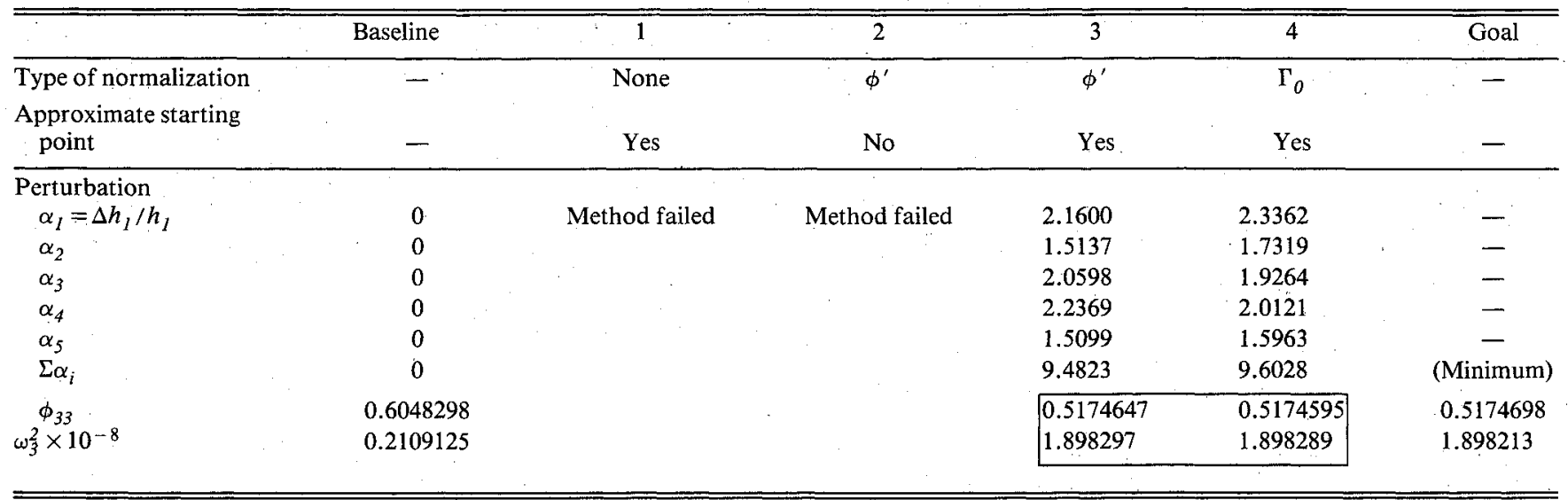

Table 6 Case 3: Frequencies of perturbed beam (subcase 3)

\begin{tabular}{lccccc}
\hline \hline Mode & 1 & 2 & 3 & 4 & 5 \\
\hline$\omega \times 10^{-2}$ & 2.6079 & 16.351 & 45.925 & 90.725 & 150.58 \\
$\omega^{\prime} \times 10^{-2}$ & 7.7532 & 51.915 & 137.78 & 261.89 & 427.56 \\
$\Delta \omega / \omega, \% 0$ & 197.30 & 217.50 & 200.00 & 188.67 & 183.94 \\
\hline \hline
\end{tabular}

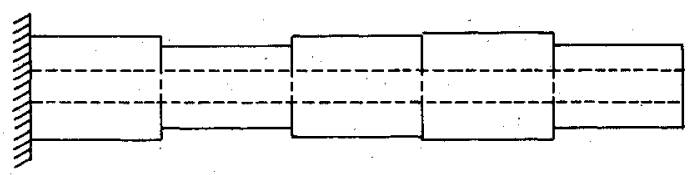

Fig. 6 Case 3 (subcase 3): redesign for change in third-mode shape and $200 \%$ increase in third frequency.

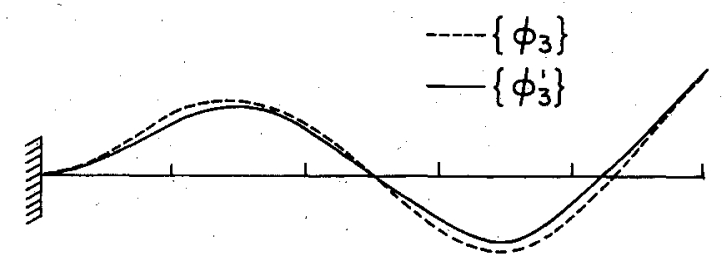

Fig. 7 Case 3 (subcase 3): third-mode shape of perturbed beam.

drift along with the perturbed frequency, with little tendency to cross.

\section{Case 3: Mode Changed, 200\% Frequency Change}

Consider a change in the third mode and a very large change in the third natural frequency:

$$
\begin{gathered}
\Delta \phi_{33}=-0.08736 \quad(-14.4 \%) \\
\Delta \lambda_{3} / \lambda_{3}=8.00 \quad(+200 \% \text { in frequency })
\end{gathered}
$$

Only the subcases involving normalization and an approximate starting point give reasonable results (Table 5). The approximate starting point used in this paper included an eigenvector expansion of the baseline modes and $\alpha_{e}$ values chosen arbitrarily at 2.0 .

Both. methods of normalization give excellent results for mode and frequency redesign. The gradient version involving $\Gamma_{0}$ provides a minimum weight about $1 \%$ superior to the version involving $\left\{\phi^{\prime}\right\}_{3}$ [Eq. (18)] after five cycles in the conjugate gradient method. The difference is likely not worth the additional complexity of $\Gamma_{0}$ in large problems.

The convergence of the method for values of $\alpha_{e}$ larger than 1 is theoretically significant. Methods based on Taylor series expansions will fail for such cases because the radius of convergence in $\alpha_{e}$ is unity.
The redesigned beam is shown in Fig. 6. Reanalysis of the perturbed beam yields the mode shape in Fig. 7 and the frequencies in Table 6. Again, the unconstrained frequencies drift with the constrained frequency with little tendency to cross.

\section{Conclusions}

A nonlinear perturbation method using a penalty function approach and conjugate gradient solver has proved to work quite well for dynamic modal redesign. Examples have involved small, moderate, and large changes and solutions have proved to be accurate when proper normalization of the error terms is used. The method has been discussed for one mode only, but the extension to more than one mode is obvious; the normalized force unbalance for each mode must appear in the penalty terms.

The method converges for large values of the design parameters $\alpha_{e}$. One example shows convergence beyond the radius of convergence for Taylor series methods; thus, the present method is felt to be more powerful than the Taylor series methods.

The method is efficient for redesign because it involves only a single finite element analysis. Competing methods often require updating of the baseline structure through finite element reanalysis.

The nonlinear perturbation method requires a normalization of the error term (force unbalance). One method, involving a gradient of the error with respect to the design variables, is very accurate but intricate and expensive in computer resources. Another method, involving the specific mode shape, is practical and almost as accurate as the gradient procedure. Normalization by such a mode is recommended.

When large changes are desired, it is helpful to use an approximate starting point for the design variables and the unconstrained modal displacements. The linear theory ${ }^{9}$ or physical intuition can provide the approximate structural design variables. Linear theory 9 or "curve fitting" procedures such as the least square error method can provide approximate unconstrained modal displacements. For modest changes, up to $30 \%$, the conjugate gradient solver can be started with zero (baseline) values for the variables and proper convergence occurs.

Rather than using a minimum weight criterion, one could alternatively use minimum structural change (from the baseline design). The objective function is then taken as

$$
f\left(\alpha_{e}\right) \equiv \sum_{e=1}^{L} \alpha_{e}^{2}
$$

which can be implemented easily.

The penalty parameter $\mu$ is chosen to equally emphasize minimum weight (or least change) and minimum error in 
nodal energy. For the present 10 degrees-of-freedom problem, $\mu$ was initially taken as 10,000 and was increased by a factor of 10 at each cycle of conjugate gradient solution. (The increasing value is needed as the error term is driven to zero.) For problems with more degrees of freedom, $\mu$ should be scaled so that the product of $\mu$ and the error term $P$ is of the same order as the expected value of $\Sigma \alpha_{e}$ (or $\Sigma \alpha_{e}^{2}$ ).

Because the redesign problem involves an inversion of the dynamic relations, the relatively placid linear vibration problem can become ill-posed (a unique inverse may not exist). When many constraints are specified, one must be careful not to choose conflicting or unreasonable physical requirements. For example, it may be difficult to increase the third frequency by $100 \%$ and decrease the first frequency by $30 \%$ in a structural system. Other overly ambitious redesigns could cause intricate crossings of unconstrained frequencies. When frequencies are close and unwanted mode shifting is expected, the key frequencies and mode shapes should be specified to prevent the crossing. In the example studied, however, mode crossing was not a problem when only one frequency was specified.

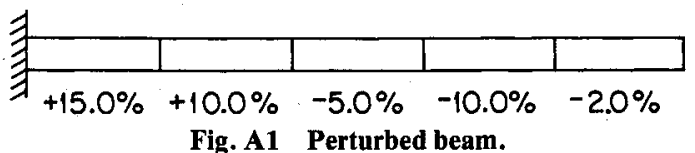

Table A1 Third mode of perturbed beam

\begin{tabular}{lccr}
\hline \multicolumn{2}{c}{ Translation } & \multicolumn{2}{c}{ Rotation } \\
\hline$\phi_{3,1}^{\prime}$ & 0 & $\phi_{3,2}^{\prime}$ & 0 \\
$\phi_{3,3}^{\prime}$ & 0.5174685 & $\phi_{3,4}^{\prime}$ & -0.003034136 \\
$\phi_{3,5}^{\prime}$ & 0.5756006 & $\phi_{3,6}^{\prime}$ & 0.002847634 \\
$\phi_{3,7}^{\prime}$ & -0.4415345 & $\phi_{3,8}^{\prime}$ & 0.004874386 \\
$\phi_{3,9}^{\prime}$ & -0.4736828 & $\phi_{3,10}^{\prime}$ & -0.005105421 \\
$\phi_{3,11}^{\prime}$ & 1.0000000 & $\phi_{3,12}^{\prime}$ & -0.008265179 \\
& $\left(\omega_{3}\right)^{2}=2.118426 \times 10^{7}(\mathrm{rad} / \mathrm{s})^{2}$ \\
\hline
\end{tabular}

One of the most difficult problems in the mathematical programming technique is the number of the free variables, i.e., the design variables and the unspecified degrees of freedom. Optimal control theory shows that the possible size for the general nonlinear problem is of the order $10^{2}$ and for the nonlinear structural problem, $10^{3}$. However, the cost of the solution should be considered. An exact optimum at much higher cost may be less desirable than a near optimum.

A reduction scheme such as Guyan's reduction may be used to reduce the size of the problem. MSC/NASTRAN provides a dynamic condensation scheme, generalized dynamic reduction. ${ }^{14,15}$ It is a good candidate for the reduction in size of dynamic systems.

The current method is being extended to include generalized dynamic reduction and to include plate elements. An industrial die casting with 1200 degrees of freedom will be redesigned as a case study for improving the algorithm. Because many optimization methods have not been adopted by industry, it is hoped that implementation of this method in MSC/NASTRAN will make it more generally accepted.

\section{Appendix: First-Order Inverse Perturbation Method}

\section{Forward Perturbation}

If one assumes that the structural changes are small, the changes in frequencies and mode shapes can be expected to be small. Hence the second- and higher-order terms in Eq. (5b) could at first thought be neglected. The first-order equation of the perturbed system is

$$
[\Delta k][\phi]+[k][\Delta \phi]=[\Delta m][\phi][\lambda]+[m][\Delta \phi][\lambda]+[m][\phi][\Delta \lambda]
$$

Table A4 Results of inverse perturbation

\begin{tabular}{lcccrc}
\hline \hline Element & $\alpha_{1}$ & $\alpha_{2}$ & $\alpha_{3}$ & $\alpha_{4}$ & $\alpha_{5}$ \\
\hline Exact \%0 & +15.0 & +10.0 & -5.0 & -10.0 & -2.0 \\
Eq. (A1) & +22.4 & +18.3 & +2.9 & -3.4 & +7.6 \\
Eq. (A2) & +17.3 & +10.9 & -4.9 & -9.0 & -2.0 \\
\hline
\end{tabular}

Table A2 First-order terms and truncation error

\begin{tabular}{crrrrrr}
\hline \hline DOF & {$[\Delta k]\left\{\phi_{3}\right\}$} & {$[k]\left\{\Delta \phi_{3}\right\}$} & {$[\Delta m]\left\{\phi_{3}\right] \lambda_{3}$} & {$[m]\left\{\Delta \phi_{3}\right\} \lambda_{3}$} & \multicolumn{1}{c}{$[m]\left\{\phi_{3}\right\} \Delta \lambda_{3}$} & \multicolumn{1}{c}{ Error ${ }^{\mathrm{a}}$} \\
\hline 1 & $4.76 \times 10^{7}$ & $-3.14 \times 10^{7}$ & $1.05 \times 10^{7}$ & $-1.09 \times 10^{7}$ & $3.95 \times 10^{5}$ & $1.44 \times 10^{7}$ \\
2 & $7.87 \times 10^{8}$ & $-2.88 \times 10^{8}$ & $-6.98 \times 10^{7}$ & $1.79 \times 10^{7}$ & $-4.95 \times 10^{6}$ & $5.53 \times 10^{8}$ \\
3 & $-8.22 \times 10^{6}$ & $1.74 \times 10^{7}$ & $4.58 \times 10^{6}$ & $5.85 \times 10^{6}$ & $3.29 \times 10^{5}$ & $-1.56 \times 10^{6}$ \\
4 & $3.68 \times 10^{9}$ & $-4.07 \times 10^{9}$ & $2.11 \times 10^{8}$ & $-3.30 \times 10^{8}$ & $6.98 \times 10^{6}$ & $-2.80 \times 10^{8}$ \\
5 & $8.52 \times 10^{6}$ & $3.03 \times 10^{6}$ & $5.66 \times 10^{6}$ & $4.17 \times 10^{6}$ & $-2.84 \times 10^{5}$ & $2.00 \times 10^{6}$ \\
6 & $-1.32 \times 10^{9}$ & $1.78 \times 10^{9}$ & $-1.57 \times 10^{8}$ & $3.40 \times 10^{8}$ & $6.41 \times 10^{6}$ & $2.69 \times 10^{8}$ \\
7 & $3.98 \times 10^{6}$ & $-1.27 \times 10^{7}$ & $4.70 \times 10^{6}$ & $-1.04 \times 10^{7}$ & $-2.17 \times 10^{5}$ & $-2.80 \times 10^{6}$ \\
8 & $2.17 \times 10^{9}$ & $-2.81 \times 10^{9}$ & $1.70 \times 10^{8}$ & $-4.58 \times 10^{7}$ & $-8.43 \times 10^{6}$ & $-7.52 \times 10^{8}$ \\
9 & $-2.60 \times 10^{6}$ & $-4.77 \times 10^{4}$ & $-8.84 \times 10^{5}$ & $-1.98 \times 10^{6}$ & $1.95 \times 10^{5}$ & $2.45 \times 10^{4}$ \\
10 & $-6.21 \times 10^{7}$ & $-4.36 \times 10^{7}$ & $-2.11 \times 10^{7}$ & $-8.79 \times 10^{7}$ & $4.65 \times 10^{6}$ & $-1.27 \times 10^{6}$ \\
\hline \hline
\end{tabular}

\footnotetext{
${ }^{a}$ Truncation error of first-order perturbation equation.
}

Table A3 Second- and higher-order terms and residual error

\begin{tabular}{crrrrrr}
\hline \hline DOF & {$[\Delta k]\left\{\Delta \phi_{3}\right\}$} & {$[\Delta m]\left\{\Delta \phi_{3}\right\} \lambda_{3}$} & {$[m]\left\{\Delta \phi_{3}\right\} \Delta \lambda_{3}$} & {$[\Delta m]\left\{\phi_{3}\right\} \Delta \lambda_{3}$} & {$[\Delta m]\left\{\Delta \phi_{3}\right\} \Delta \lambda_{3}$} & Error $^{\mathrm{a}}$ \\
\hline 1 & $-1.58 \times 10^{7}$ & $-1.35 \times 10^{6}$ & $-4.82 \times 10^{4}$ & $4.65 \times 10^{4}$ & $-5.97 \times 10^{3}$ & $-1.02 \times 10^{2}$ \\
2 & $-5.61 \times 10^{8}$ & $-5.50 \times 10^{6}$ & $7.90 \times 10^{4}$ & $-3.08 \times 10^{5}$ & $-2.43 \times 10^{4}$ & $-5.32 \times 10^{3}$ \\
3 & $1.16 \times 10^{6}$ & $-4.48 \times 10^{5}$ & $2.58 \times 10^{4}$ & $2.02 \times 10^{4}$ & $-1.97 \times 10^{3}$ & $-8.90 \times 10^{0}$ \\
4 & $2.83 \times 10^{8}$ & $3.74 \times 10^{6}$ & $-1.45 \times 10^{6}$ & $9.30 \times 10^{5}$ & $1.65 \times 10^{4}$ & $2.07 \times 10^{3}$ \\
5 & $-2.07 \times 10^{6}$ & $-1.12 \times 10^{5}$ & $1.84 \times 10^{4}$ & $2.50 \times 10^{4}$ & $-4.92 \times 10^{2}$ & $2.27 \times 10^{2}$ \\
6 & $-2.91 \times 10^{8}$ & $-2.25 \times 10^{7}$ & $1.50 \times 10^{6}$ & $-6.92 \times 10^{5}$ & $-9.93 \times 10^{4}$ & $3.61 \times 10^{3}$ \\
7 & $3.46 \times 10^{6}$ & $6.77 \times 10^{5}$ & $-4.59 \times 10^{4}$ & $2.07 \times 10^{4}$ & $2.98 \times 10^{3}$ & $8.90 \times 10^{1}$ \\
8 & $7.68 \times 10^{8}$ & $1.50 \times 10^{7}$ & $-2.02 \times 10^{5}$ & $7.49 \times 10^{5}$ & $6.64 \times 10^{4}$ & $3.22 \times 10^{3}$ \\
9 & $2.80 \times 10^{3}$ & $3.97 \times 10^{4}$ & $-8.75 \times 10^{3}$ & $-3.90 \times 10^{3}$ & $1.75 \times 10^{2}$ & $1.44 \times 10^{2}$ \\
10 & $2.56 \times 10^{6}$ & $1.76 \times 10^{6}$ & $-3.88 \times 10^{5}$ & $-9.31 \times 10^{4}$ & $7.76 \times 10^{3}$ & $9.09 \times 10^{3}$ \\
\hline \hline
\end{tabular}

\footnotetext{
${ }^{a}$ Residual error of full nonlinear perturbation equation.
} 
where the terms for the baseline system, $[k][\phi]$ and $[m][\phi][\lambda]$ are eliminated.

A forward perturbation is done to test the accuracy of the first-order equation. The baseline structure is a cantilever Euler-Bernoulli beam perturbed in thickness by the amounts shown in Fig. A1. The perturbed frequencies and mode shapes are obtained using the inverse power method in MSC/NASTRAN. Table A1 shows the resulting third mode of the perturbed beam. The perturbations in stiffness matrix, mass matrix, mode shapes, and frequencies are extracted through the use of DMAP.

When these "exact" perturbations for the third mode are inserted into Eq. (A1), the truncation error is of the same order as the terms in the equation, as shown in Table A2. To complete the comparison, nonlinear terms from Eq. (5b) are shown in Table A3 and point out the importance of the $[\Delta k][\Delta \phi]$ terms in such a typical problem.

\section{Inverse Perturbation}

The inverse perturbation procedure is applied to test the accuracy of the first-order equation by using the "exact" values of $[\Delta \phi],[\Delta \lambda]$ calculated from the forward perturbation. Again, only the third mode is considered.

Since $[\Delta \phi],[\Delta \lambda]$ are known, the first-order equation (A1) becomes overdetermined. To obtain the values of the design variables $\alpha_{i}$, the least square method was used. Results are given in Table A4, showing substantial errors in the inverse process.

In the above subsection, it was shown that one of the second-order terms, $[\Delta k][\Delta \phi]$ is as large as the first-order terms. If we include $[\Delta k][\Delta \phi]$, the perturbation equation becomes

$$
\begin{aligned}
& {[\Delta k][\phi]+[k][\Delta \phi]+[\Delta k][\Delta \phi]} \\
& \quad=[\Delta m][\phi][\lambda]+[m][\Delta \phi][\lambda]+[m][\phi][\Delta \lambda]
\end{aligned}
$$

This modified equation has been solved for $\alpha_{i}$, again with "exact" $[\Delta \phi]$ and $[\Delta \lambda]$, and yields results in Table A4.

Both of the solutions presented in Table A4 contain the linear approximation

$$
[\Delta k]_{e}=[k]_{e}(3 \Delta h / h)_{e}
$$

rather than the exact cubic relation between thickness change and moment of inertia. This accounts for an error of up to $5 \%$ in both solutions.
The linearized solution Eq. (A1) is not sufficiently accurate for this sample problem. The modified equation (A2) has error that could be acceptable for some cases.

\section{Acknowledgment}

This research was supported by NSF Research Grant MEA8019642, "Inverse Perturbation in Finite Element Dynamic Analysis."

\section{References}

${ }^{1}$ Stetson, K. A., "Perturbation Method of Structural Design Relevant to Holographic Vibration Analysis," AIAA Journal, Vol. 13, April 1975, pp. 457-459.

${ }^{2}$ Stetson, D. A. and Palma, G. E., "Inversion of First Order Perturbation Theory and its Application to Structural Design," AIAA Journal, Vol. 14, April 1976, pp. 454-460.

${ }^{3}$ Stetson, K. A., Harrison, I. R., and Palma, G. E., "Redesigning Structural Vibration Modes by Inverse Perturbation Subject to Minimal Change Theory," Computer Methods in Applied Mechanics and Engineering, Vol. 16, 1978, pp. 151-175.

${ }^{4}$ Stetson, K. A. and Harrison, I. R., "A Study of Structural Redesign by Finite-Element Inverse Perturbation of Vibration Modes," United Technologies Research Center, Rept. R80-191942-1, Jan. 1980.

${ }^{5}$ Pierson, B. L., "An' Optimal Control Approach to MinimumWeight Vibrating Beam Design," Journal of Structural Mechanics, Vol. 5, No. 2, 1977, pp. 147-178.

${ }^{6}$ Schmit, L. A., "Structural Synthesis-Its Genesis and Development," AIAA Journal, Vol. 19, Oct. 1981, pp. 1249-1263.

${ }^{7}$ Vanderplaats, G. N., "Structural Optimization-Past, Present, and Future," AIAA Journal, Vol. 20, July 1982, pp. 992-1000.

${ }^{8}$ Bathe, K. J. and Wilson, E. L., Numerical Methods in Finite Element Analysis, Prentice Hall, Englewood Cliffs, N.J., 1976, pp. 370-371, 495-496.

${ }^{9}$ Sandstrom, R. E. and Anderson, W. J., "Modal Perturbation Method for Marine Structure,'” Paper presented at Annual Meeting of Society of Naval Architects and Marine Engineers, Nov. 1982 (see also Transactions of the Society of Naval Architects and Marine Engineers, Vol. 90, 1982, pp. 41-54).

${ }^{10}$ Kirsch, U., Optimum Structural Design, McGraw-Hill Book Co., New York, 1981.

${ }^{11}$ Haug, E. J. and Arora, J. S., Applied Optimal Design, John Wiley \& Sons, New York, 1979.

${ }^{12}$ Luenberger; D. G., Introduction to Linear and Nonlinear Programming, Addison-Wesley, Reading, Mass., 1973.

13 "IBM System 360 Scientific Subroutine Package, Version III, Programmers Manual," 5th ed., International Business Machines Corp., New York, Aug. 1970.

${ }^{14}$ Schaeffer, H. G., "MSC/NASTRAN PRIMER," Schaeffer Analysis, Inc., Mount Vernon, N.H., 1979.

15 “MSC/NASTRAN Applications Manual 2.4," MacNealSchwendler Corp., Los Angeles, Calif., April 1981. 